\title{
Zonulysis during Femtosecond Laser-Assisted Cataract Surgery
}

\section{Dear Editor,}

Femtosecond laser assisted cataract surgery (FLACS) has gained popularity globally with increasing number of surgeons being familiar with the procedure. ${ }^{1}$ Despite an initial learning curve, ${ }^{2}$ it is non-inferior to standard phacoemulsification, ${ }^{3}$ and is beneficial in reducing endothelial loss and improving intraocular lens (IOL) centration in selected patients. ${ }^{4}$

However, despite its purported benefits, complications similar to standard phaco-emusification are known to occur, including posterior capsule rupture, vitreous loss and dropped nucleus. ${ }^{5}$ Furthermore, there are unique risks of laser application to the cornea ${ }^{6}$ iris injury and incomplete capsular tags, which may occur with improper centration, or loss of vacuum.

In our tertiary institution, we perform over 300 cases of FLACS per year. In this report, we describe our experience in dealing with intra-operative zonulysis in the early stages of cataract surgery of 3 eyes following femtosecond laser assisted capsulorrhexis creation and lens fragmentation with the Catalys ${ }^{\mathrm{TM}}$ (Optimedica) in seemingly normal eyes. Each patient had surgery performed by a different surgeon, using a similar technique.

We present 3 patients with seemingly normal eyes apart from visually significant cataracts during preoperative assessment.

Patient 1 is a 78 -year-old Malay man, with best corrected visual acuities (BCVA) of 20/20 on the right (pseudophakic) and 20/400 on the left, with moderately dense nuclear sclerotic cataract.

Patient 2 is a 75-year-old Malay man with BCVA was 20/25 on the right (pseudophakic) and 20/50 on the left, with a nuclear sclerotic cataract.

Patient 3 is a 77-year-old Chinese woman, BCVA was $20 / 70$ on the right and 20/50 on the left, with a macular potential 20/30 in both eyes. She had bilateral nuclear sclerotic cataracts.

All three patients had successful completion of femtosecond laser assisted capsulorrhexis creation and nucleus fragmentation with Catalys ${ }^{\mathrm{TM}}$ under topical anaesthesia. There were no capsular tags noted, and no additional force was required to remove the anterior capsule. Following removal of the anterior capsule, each surgeon then gently balloted the lens and was able to easily release the air bubble, before performing hydrodissection of the nucleus. However, while attempting to crack the nucleus after creating a central trough, zonulysis of approximately $4-5$ clock hours was observed and there was asymmetry in the anterior chamber depth. All 3 patients required conversion to intracapsular cataract extraction (ICCE) and anterior chamber intraocular lens (ACIOL) implantation. Insertion of a device such as capsular tension ring was not attempted to avoid further intraocular manipulation that could result in nucleus drop (Fig. 1).

Post-operatively, all 3 patients were closely followed up to monitor for complications. Patient 1 was noted to have vitreous wick extending at 3 clock hours, with no posterior segment complications. At post-operative month 1, he underwent YAG vitreolysis and subsequently had an uneventful recovery. At postoperative month 6 , his uncorrected visual acuity (UCVA) was 20/25, and he was satisfied with his surgical outcome.

Patient 2 had a superior descemet membrane (DM) detachment extending from the main wound, away from his visual axis. He was counselled for air bubbling to re-attach the DM, which was performed within two weeks from initial surgery. At post-operative month 6 , his BCVA was 20/30.

Patient 3 had a vitreous haemorrhage, which was conservatively managed and followed closely to watch for retinal tears and detachment. The haemorrhage resolved spontaneously over time without intervention. At post-operative month 6, her BCVA was 20/30 .

The introduction of FLACS has the potential of increasing surgical precision, ${ }^{7}$ with accurate wound incisions and reliable capsulotomies, allowing precise IOL centration. It has also been shown to reduce the effective phacoemulsification time (EPT) ${ }^{8}$ which aims to reduce endothelial cell loss, ${ }^{9}$ wound burns and postoperative inflammation.

Even though the technological advancement to incorporate femtosecond laser as part of cataract surgery were initially encouraging, the risk of intra-laser, 


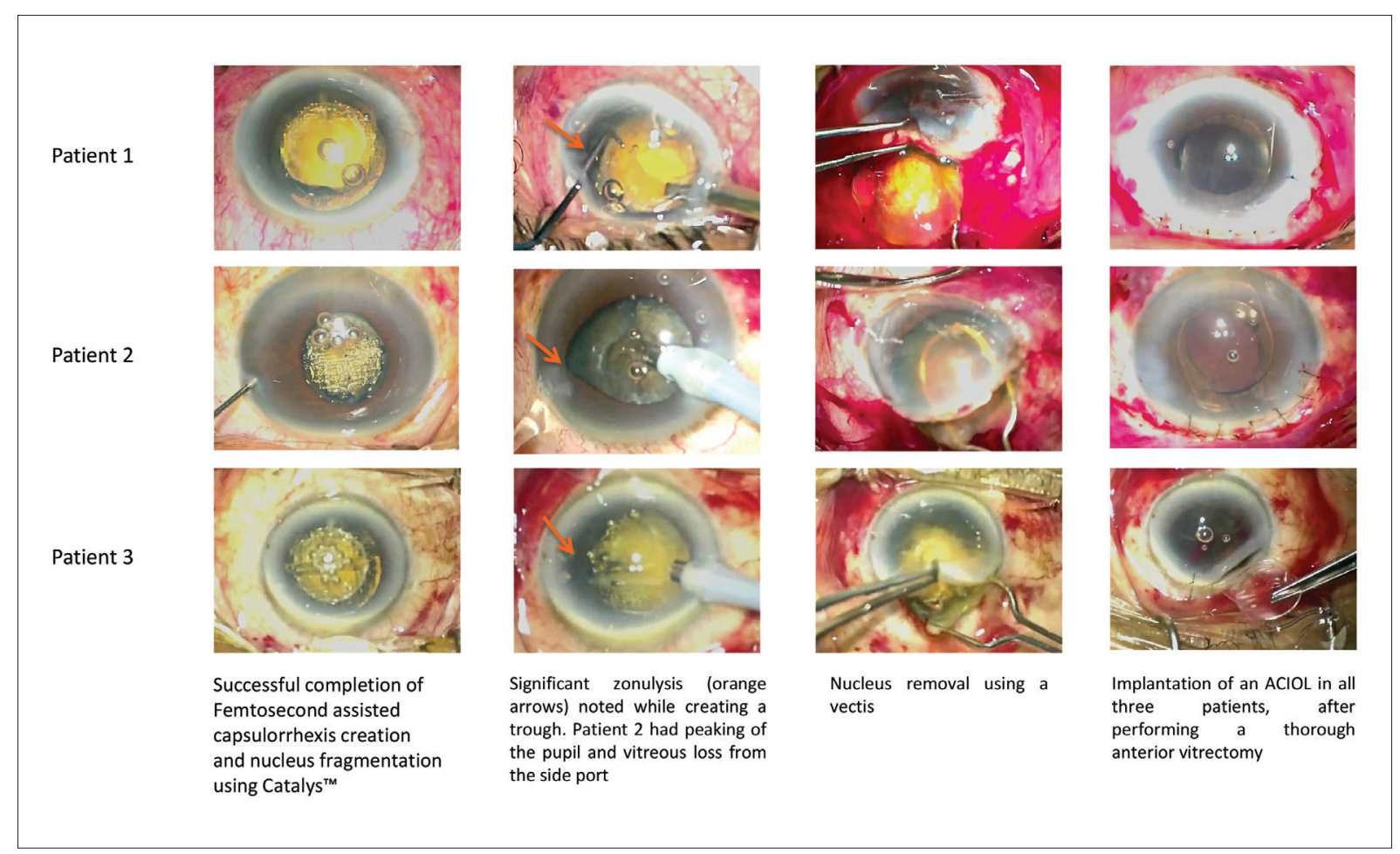

Fig. 1. All 3 patients suffered significant zonulysis early in the course of surgery, which required conversion to an intracapsular cataract extraction (ICCE) and anterior chamber intraocular lens (ACIOL) implantation.

intraoperative and post-operative complications have yet to be overcome. In particular, post-laser miosis, incomplete capsulotomy and anterior capsular tears ${ }^{10}$ are specific to the use of femtosecond laser. Post-operative cystoid macular oedema has been noted to be more significant after FLACS compared to conventional phacoemulsification. ${ }^{11}$

Photodisruption by the femtosecond laser produces micro-cavitation bubbles, which expand to form a resection plane as part of nucleus fragmentation. During the early stages of surgery, it has been recommended that the air bubbles be released to reduce pressure within the lens, and shallowing of the anterior chamber.

Contrary to previously published articles on use of FLACS in adult and paediatric patients with known subluxed cataracts ${ }^{12-14}$ all 3 patients in this case series had neither clinical evidence nor risk factors for zonulysis prior to cataract surgery. Chee et al. reported use of FLACS in carefully selected patients with at least 6 clock hours of known zonulysis. In that series, femtosecond laser was applied with a different platform and fragmentation pattern. In addition, anterior vitrectomy, capsular tension ring segments and capsular hooks were employed prior to nucleus removal in some patients. Minimal or no hydro-dissection was performed, and gas bubbles that were not obviously visible, were not released. ${ }^{12}$ This is in contrast to our patients, for which all three surgeons had performed the steps of surgery that were recommended.

In our patients, we postulate that there was likely preexisting, mild and clinically unapparent zonulysis, which could have been exaggerated following femtosecond laser completion due to the sudden expansion of gas causing downward displacement of the entire capsular bag, possibly damaging more zonules. During balloting to release the air bubbles, these compromised zonules lose their integrity, leading to gross zonulysis and tilting of the lens.

Each of our patients experienced different postoperative complications within the first month of surgery. Fortunately, all 3 patients were managed appropriately and attained good BCVA by 6 months.

Our study is limited by the absence of intra-operative imaging to prove the presence of lens tilt following FLACS. With advances in intra-operative anterior segment imaging for cornea and cataract surgeries, ${ }^{15}$ we could potentially utilise intra-operative optical coherence tomography in future to demonstrate this hypothesis, which currently is speculative.

In conclusion, as more surgeons become familiar with performing FLACS, and with greater demand for the procedure by our patients, surgeons should be aware 
of the learning curve and challenges involved. We hope that by sharing our experience, this would alert other surgeons to potential problems they may encounter while performing FLACS.

\section{REFERENCES}

1. Tam YS, Kumar CM, Au Eong KG, et al. Trends in cataract surgery technique and anaesthesia preferences in Singapore: a 2016 survey. Ann Acad Med Singap 2018;47:390-3.

2. Christy JS, Nath M, Mouttapa F, et al. Learning curve of femtosecond laser-assisted cataract surgery: experience of surgeons new to femtosecond laser platform. Indian J Ophthalmol 2017;65:683-9.

3. Mursch-Edlmayr AS, Bolz M, Luft N, et al. Intraindividual comparison between femtosecond laser-assisted and conventional cataract surgery. J Cataract Refract Surg 2017;43:215-22.

4. Alio JL, Soria F, Abdou AA. Femtosecond laser assisted cataract surgery followed by coaxial phacoemulsification or microincisional cataract surgery: differences and advantages. Curr Opin Ophthalmol 2014;25:81-8.

5. YeZ, LiZ, He S. Ameta-analysis comparing postoperative complications and outcomes of femtosecond laser-assisted cataract surgery versus conventional phacoemulsification for cataract. J Ophthalmol 2017;2017:3849152.

6. Chai CHC, Sundar G. Inadvertent corneal laser application in femtosecond laser-assisted cataract surgery. JCRS Online Case Reports 2016;4:45-8

7. Nagy Z, Takacs A, Filkorn T, et al. Initial clinical evaluation of an intraocular femtosecond laser in cataract surgery. J Refract Surg 2009;25:1053-106.

8. Huseynova T, Mita M, Corpuz CC, et al. Evaluating the different laser fragmentation patterns used in laser cataract surgeries in terms of effective phacoemulsification time and power. Clin Ophthalmol 2015; 9:2067-71.
9. Conrad-Hengerer I, Al Juburi M, Schultz T, et al. Corneal endothelial cell loss and corneal thickness in conventional compared with femtosecond laser-assisted cataract surgery: three-month follow-up. J Cataract Refract Surg 2013;39:1307-13.

10. Roberts TV, Lawless M, Bali SJ, et al. Surgical outcomes and safety of femtosecond laser cataract surgery: a prospective study of 1500 consecutive cases. Ophthalmology 2013;120:227-33.

11. Hooshmand J, Vote BJ. Femtosecond laser assisted cataract surgery, technology, outcome, future directions and modern applications. Asia Pac J Ophthalmol (Phila) 2017;6:393-400.

12. Chee SP, Wong MH, Jap A. Management of severely subluxated cataracts using femtosecond laser-assisted cataract surgery. Am J Ophthalmol 2017;173:7-15.

13. Crema AS, Walsh A, Yamane IS, et al. Femtosecond laser-assisted cataract surgery in patients with Marfan syndrome and subluxated lens. J Refract Surg 2015;31:338-41.

14. Teshigawara T, Meguro A, Sanjo S, et al. The advantages of femtosecond laser-assisted cataract surgery for zonulopathy. Int Med Case Rep J 2019;12:109-16.

15. Titiyal JS, Kaur M, Falera R. Intraoperative optical coherence tomography in anterior segment surgeries. Indian J Ophthalmol 2017;65:116-21.

Hazel A Lin, ${ }^{1}$ MBBS, MMed (Ophth),

Clement WT $\underline{\text { Tan }},{ }^{1}$ MBBS, MMed (Ophth), FRCSEd,

Cheryl S $\underline{\text { Ngo }}$, ${ }^{1}$ MBBS, MMed (Ophth), FRCSEd

${ }^{1}$ Department of Ophthalmology, National University Hospital, Singapore

Address for Correspondence: Dr Hazel Anne Lin Hui'En, Department of Ophthalmology, National University Hospital, 1E Kent Ridge Road, Singapore 119228.

Email: hazel_anne_lin@nuhs.edu.sg 